ISSN 1409-2441

www.ucr.ac.cr
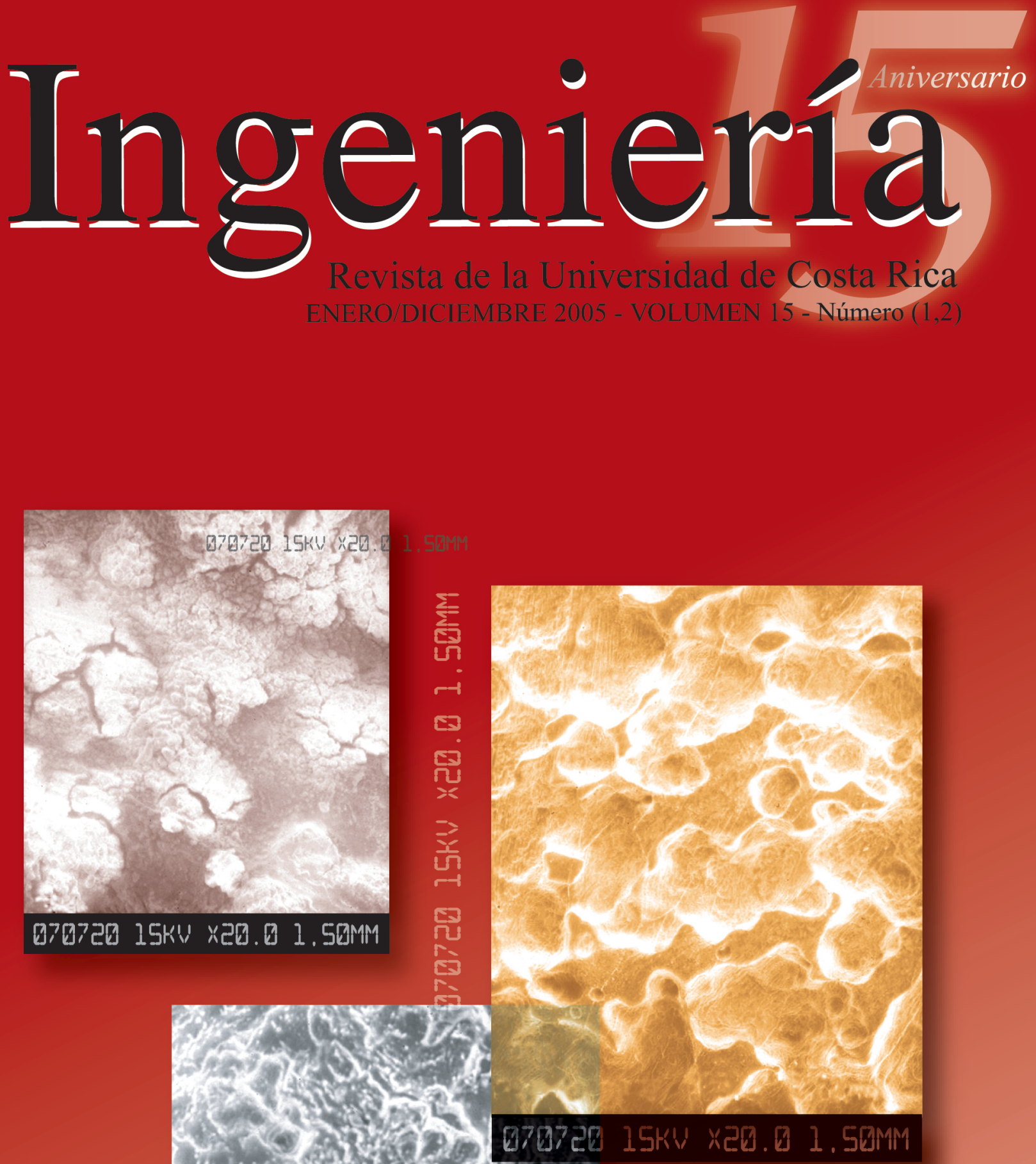

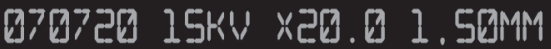

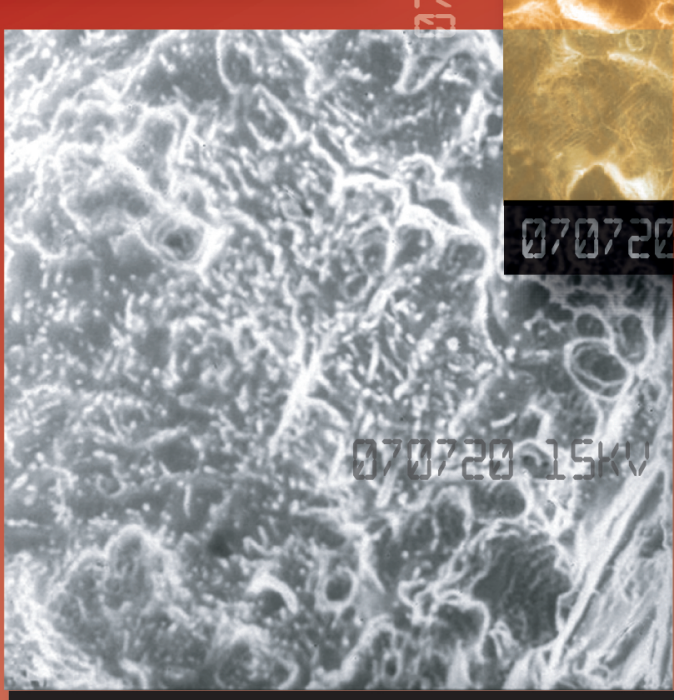

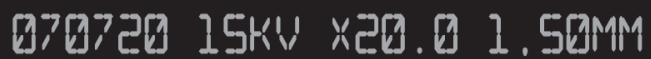




\title{
EVALUACIÓN DE ALEACIONES DE MAGNESIO PARA ÁNODOS GALVÁNICOS
}

\author{
Mario Alberto Vega León
}

\begin{abstract}
Resumen
Se caracterizan tres aleaciones comerciales de magnesio para ánodos galvánicos mediante análisis químico, ensayos electroquímicos e inspección microscópica.

La evaluación electroquímica se llevó a cabo por medio de pruebas galvanostáticas para determinar las pérdidas por disolución. Se midieron sus potenciales de circuito abierto y cerrado; además se realizaron inspecciones microscópicas (metalográfico y electrónico de barrido) de los materiales y sus productos de corrosión. Por último, se obtuvo su capacidad de drenaje de corriente y eficiencia y se proponen una serie de ensayos como guía para comparar el desempeño de las aleaciones estudiadas.
\end{abstract}

Se sugiere experimentar con otras aleaciones tales como zinc y aluminio que puedan ser utilizadas en sistemas de ánodos galvánicos, para desarrollar metodologías y criterios para su control de calidad.

Palabras clave: corrosión, ánodos galvánicos, protección catódica.

\begin{abstract}
Three commercial magnesium alloys for galvanic anodes were characterized by chemical analysis, electrochemical testing and microscopy inspection.

Electrochemical evaluation was carry out by galvanostatic testing to know dissolution metal lost. Their open and close circuit potentials were recorded. Microscopic examination (optic and scanning electron) for testing material and their corrosion products was done. Drain current capacity and efficiency for each alloy were obtained. A list of tests to evaluate performance of these kind of material was proposed.
\end{abstract}

Testing zinc and aluminum alloys for galvanic systems is recommended to develop methodologies and criteria for their quality control.

Key words: corrosion, galvanic anodes, cathodic protection.

Recibido: 02 de febrero del 2005 / Aprobado: 22 de julio del 2005

\section{INTRODUCCIÓN}

En Costa Rica, se ha determinado la necesidad de contar con procedimientos para caracterizar las aleaciones de ánodos que se utilizarán en los sistemas de protección catódica, de manera que se puedan seleccionar los materiales de mejor calidad. Resulta de gran importancia, disponer de un método para la selección de aleaciones más eficientes, para ser utilizadas como ánodos en cualquier proyecto de instalación de sistemas de protección catódica. Esto se reflejará en una optimización en la inversión necesaria para la protección de estructuras enterradas o sumergidas.

La protección catódica es un método para contrarrestar el mecanismo natural de la corrosión de estructuras metálicas, que se encuentran enterradas o sumergidas. Consiste en desplazar el potencial de la superficie por proteger, por medio del suministro de una cantidad de electrones suficiente, para que tome un carácter catódico, manteniendo así la estructura inmune (González, 1989). 
Existen dos métodos para proteger catódicamente una estructura: los que se clasifican de acuerdo con el proceso con que se inyecta la corriente, estos son: el método de ánodos galvánicos o de sacrificio y el de corriente impresa. En ambos casos se descarga una corriente, mediante un sistema de electrodos, ubicados en el electrolito que rodea a la estructura.

El sistema por ánodos galvánicos o de sacrificio, consiste en aprovechar la diferencia de potencial entre el elemento que se desea proteger y un metal o aleación, que se utiliza como ánodo. Los ánodos, que deben ser de un material más activo termodinámicamente que la estructura, se instalan en el electrolito y se conectan eléctricamente a la superficie que se desea proteger. Como resultado de la diferencia de potencial, se crea una pila galvánica y fluye una corriente a través del electrolito desde el ánodo hasta la estructura; de esta forma, se tendrá desgaste del material de sacrificio, logrando que el metal de la estructura permanezca intacto.

En la protección por corriente impresa, se utiliza una fuente externa de energía para proveer el flujo de electrones necesario, para disminuir el potencial de la estructura, entregando corriente directa (DC). En el electrolito, se instalan unos ánodos de dispersión y se conectan al terminal positivo de la fuente (rectificador). La estructura por proteger se conecta al extremo negativo, forzando la corriente a fluir desde los ánodos de dispersión, hacia la estructura a través del electrolito. Este sistema se aplica, cuando el requerimiento de corriente para la protección es muy grande y los ánodos de sacrificio no son suficientes.

Acerca de los ánodos de sacrificio, se puede decir suscintamente, que no son metales puros; las aleaciones son más convenientes, ya que ciertos aleantes le confieren características que mejoran su desempeño, por ejemplo, pueden disminuir el tamaño de grano, favoreciéndose el desgaste uniforme y aumentando la corriente de salida, pueden reducir también la formación de películas pasivantes superficiales según mencionan (Von Baeckmann, Schwenk \& Prinz, 1997). Entre las principales características que deben cumplir las aleaciones para ser empleadas como ánodos. (Ávila \& Genescà, 1989) citan:

- Poseer un potencial de oxidación más alto que el metal que se protege. Esta diferencia debe ser capaz de polarizar la estructura hasta potenciales de inmunidad.

- Los óxidos del material anódico, no deben formar capas aislantes o pasivantes, que dificulten la salida de corriente a tierra.

- Su desgaste debe ser lo más bajo posible y presentarse en forma homogénea en toda la superficie.

- Tener una alta capacidad de drenar corriente $(\mathrm{A} \cdot \mathrm{h} / \mathrm{kg})$.

Entre las aleaciones más utilizadas para ánodos de sacrificio se encuentran el magnesio $(\mathrm{Mg})$, el zinc $(\mathrm{Zn})$ y el aluminio $(\mathrm{Al})$, ya que su empleo ha mostrado dar los mejores resultados (Genescá, 1993). El control de impurezas y la adición de aleantes son indispensables para lograr la composición adecuada; sin embargo, el control de calidad de la colada, su proceso de cristalización y tratamiento térmico son también puntos críticos para lograr alto desempeño.

\section{METODOLOGÍA}

Se seleccionaron tres aleaciones de $\mathrm{Mg}$ disponibles en el comercio y normalizadas por American Society of Testing Materials (ASTM, 2002), para llevar a cabo una clasificación de su eficiencia electroquímica. Dos de ellas corresponden a aleación de "alto potencial" y la tercera es del tipo AZ 63.

Se determinaron las composiciones de las muestras por medio de absorción atómica y se compararon con los estándares para estos tipos de aleaciones.

Se realizaron mediciones electroquímicas, para evaluar el drenaje de corriente y las pérdidas de material por disolución. El sistema de trabajo utilizado es el que se muestra en la Figura 1. 
Este es sugerido por la norma ASTM G-97. Las celdas consistían de cilindros de acero al carbono, conteniendo una solución electrolítica de $5 \mathrm{~g}$ de sulfato de calcio $\left(\mathrm{CaSO}_{4} \cdot \mathrm{H}_{2} \mathrm{O}\right)$ y $0,1 \mathrm{~g}$ de hidróxido de magnesio $\left(\mathrm{Mg}(\mathrm{OH})_{2}\right)$ en agua hasta $1 \mathrm{~L}$, dentro de la cual se sumergieron las muestras anódicas. Estas eran barras de sección circular de $50 \mathrm{~mm}$ de longitud y $10 \mathrm{~mm}$ de diámetro, extraídas de ánodos comerciales, las cuales fueron lavadas, secadas, desengrasadas con acetona y pesadas en balanza analítica previamente. Como coulombímetros, se emplearon recipientes de vidrio con una solución saturada de sulfato de cobre, en ésta se sumergieron dos placas de cobre paralelas y entre ellas, sin que hubiera contacto eléctrico, se colocó un alambre de cobre que había sido lijado con lija grado 600 , lavado, secado, desengrasado con acetona y pesado en la balanza analítica.

Como galvanostato se utilizó un equipo de pruebas electroquímicas Gill 8 de la casa $A C M$ Instruments, el cual se conecta a un computador, que cuenta con un programa para desarrollar los ensayos.

Las muestras fueron polarizadas galvanostáticamente, aplicándoles una corriente de $10 \mathrm{~mA}$ durante $72 \mathrm{~h}$. El potencial de circuito cerrado de las muestras se determinó a las 24 h, 48 h y 72 h, y también se midió 1 h después de finalizada la prueba, para esto se empleó un electrodo de referencia de $\mathrm{Cu} / \mathrm{CuSO}_{4}$ y un voltímetro de alta impedancia. Los ensayos se llevaron a cabo por triplicado a una temperatura de $22{ }^{\circ} \mathrm{C} \pm 5{ }^{\circ} \mathrm{C}$.

Posteriormente, se retiraron las probetas y los alambres de cobre de los coulombimétros. Las primeras, se limpiaron químicamente, sumergiéndolas durante $30 \mathrm{~min}$. Esto se hizo utilizando una solución de trióxido de cromo $\left(\mathrm{CrO}_{3}\right)$ al $25 \%$ a temperatura ambiente, luego fueron enjuagadas con agua, secadas y pesadas en balanza analítica para determinar su nueva masa. Por su parte, los alambres, fueron pasados directamente a enjuague, secado y se midió su nueva masa.

La capacidad de drenaje de corriente se determinó gravimétricamente. Para esto, se calculó la carga total circulada, obtenida mediante un coulombímetro, la cual fue multiplicada por la diferencia de masa de la muestra antes y después de la prueba y dividida entre el área expuesta de la probeta.

Se realizaron inspecciones microscópicas de las superficies para caracterizar su morfología. Para el análisis de las microestructuras de las aleaciones, se utilizó un microscopio metalográfico Olympus modelo MPE-3, con las superficies pulidas a grado espejo con Alúmina $\left(\mathrm{Al}_{2} \mathrm{O}_{3}, 0,05 \mu \mathrm{m}\right)$,

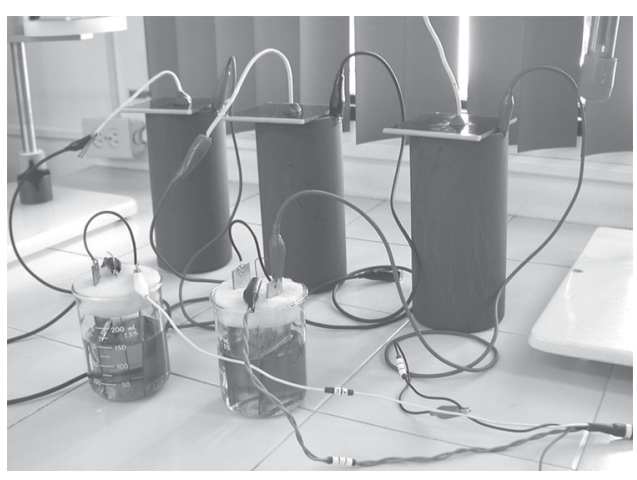

b) Detalle de las celdas

Figura 1. Sistema de trabajo. 
atacadas con solución Nital $\left(5 \% \mathrm{HNO}_{3}\right.$ en $\left.\mathrm{C}_{2} \mathrm{H}_{5} \mathrm{OH}\right)$, mientras que para los productos de corrosión y las superficies resultantes se recurrió a la técnica de microscopía electrónica de barrido (MEB), empleando un microscopio Hitachi modelo S-570.

\section{RESULTADOS}

Los resultados de los análisis químicos se muestran en el Cuadro 1, además de la composición teórica de las aleaciones, de acuerdo con los requerimientos de la norma ASTM B-843.
Una vez desarrollados los ensayos electroquímicos, se evaluó la capacidad de entrega de corriente por la técnica de gravimetría. En el Cuadro 2 , se informa la corriente promedio drenada por cada una de las aleaciones y su correspondiente eficiencia.

La microestructura de las aleaciones estudiadas se muestran en las fotomicrografías de la Figura 2. La morfología de los productos de corrosión, observados por medio de MEB se presentan en la Figura 3, mientras que en las Figuras 4 y 5 puede observarse el desgaste de la superficie por corrosión del material anódico.

Cuadro 1. Componentes encontrados en cada una da las muestras estudiadas.

\begin{tabular}{llllccc} 
& \multicolumn{2}{c}{ Muestra A } & \multicolumn{2}{c}{ Muestra B } & \multicolumn{2}{c}{ Muestra C } \\
Componente & Teórico & Real & Teórico & Real & Teórico & Real \\
& $\%$ & $\%$ & $\%$ & $\%$ & $\%$ & $\%$ \\
Manganeso (Mn) & $0,15-0,7$ & 0,19 & $0,5-1,3$ & 0,63 & $0,5-1,3$ & 0,64 \\
Aluminio (Al) & $5,0-7,0$ & 6,10 & 0,01 & 0,04 & 0,01 & 0,01 \\
Cobre $(\mathrm{Cu})$ & 0,1 & 0,07 & 0,02 & 0,03 & 0,02 & 0,002 \\
Zinc $(\mathrm{Zn})$ & $2,0-4,0$ & 3,13 & - & 0,039 & - & 0,01 \\
Níquel $(\mathrm{Ni})$ & 0,003 & 0,005 & 0,001 & 0,002 & 0,001 & 0,003 \\
Hierro $(\mathrm{Fe})$ & 0,003 & 0,014 & 0,03 & 0,017 & 0,03 & 0,006 \\
Otros & 0,30 & N.D & 0,3 & N.D & 0,3 & N.D \\
Magnesio $(\mathrm{Mg})$ & Mín. 91 & 83,5 & Mín. 98 & 91,0 & Mín. 98 & 90,0
\end{tabular}

Fuente: (El autor)

Cuadro 2. Drenaje de corriente promedio de las aleaciones.

Muestra

B

C
Corriente drenada

$(\mathbf{m A} \cdot \mathbf{h} / \mathbf{k g})$

1315
1196
1288

1315

1288

Fuente: (El autor)
Eficiencia electroquímica

60

54

58 


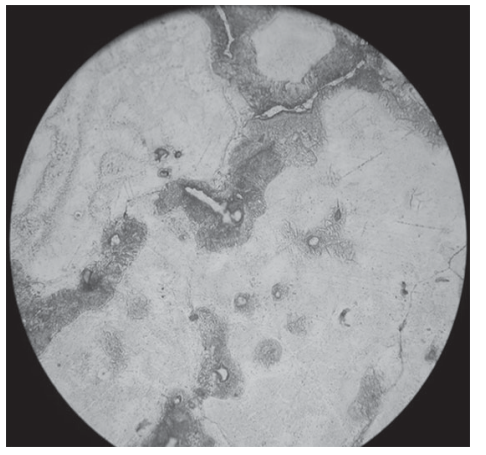

Muestra A

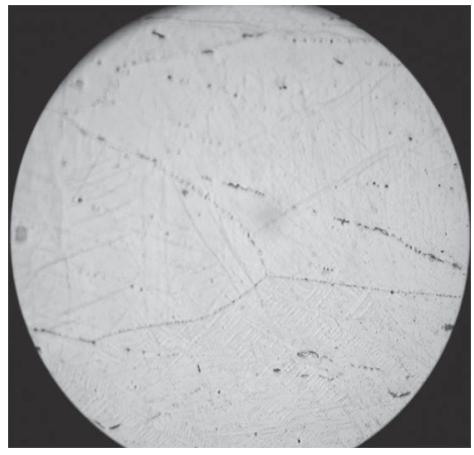

Muestra B

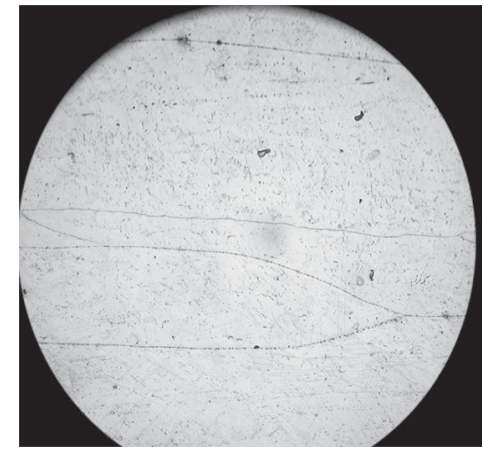

Muestra B

Figura 2. Metalografías de las muestras anódicas a 200X atacadas con Nital.

Fuente: (El autor)

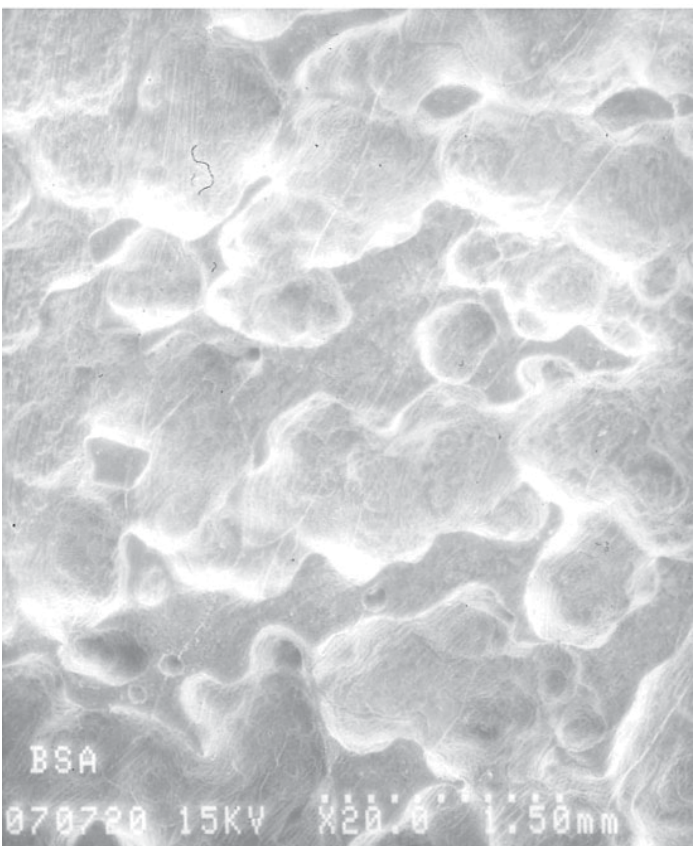

Muestra B

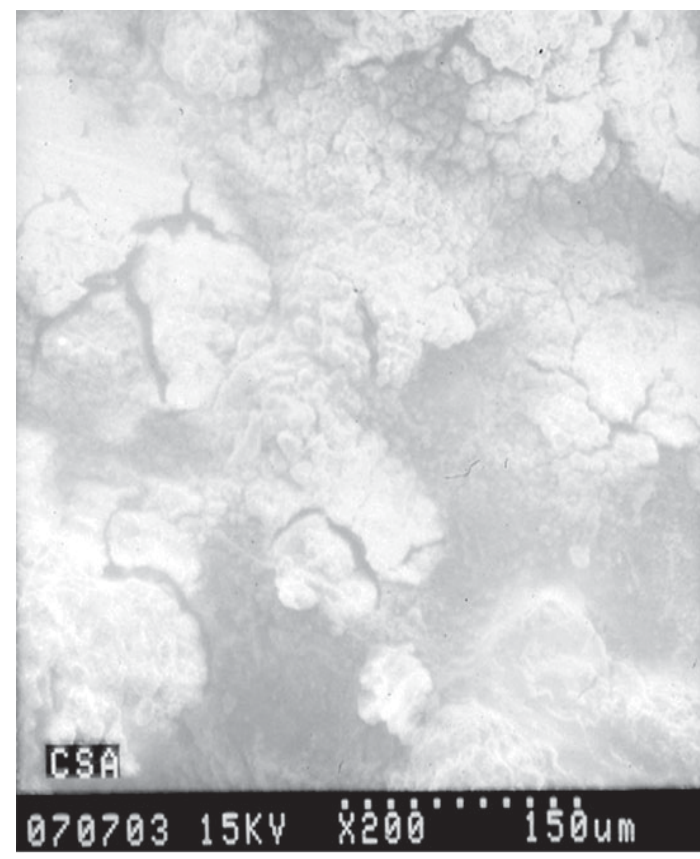

Muestra C

Figura 3. MEB de los productos de corrosión de las muestra B y C a 200X.

Fuente: (El autor) 


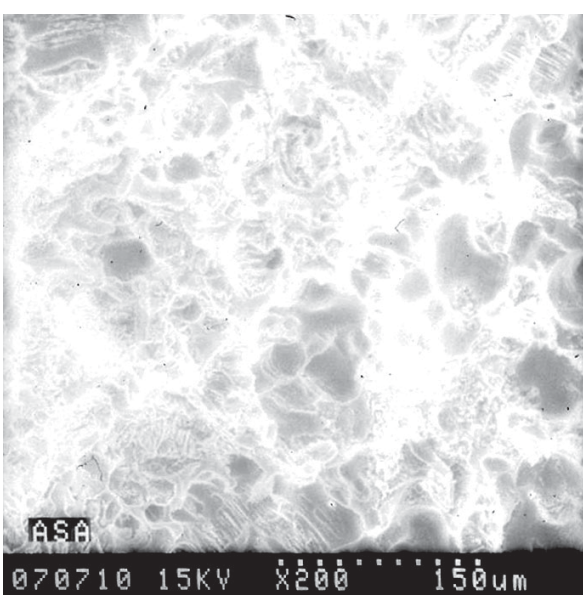

Muestra A

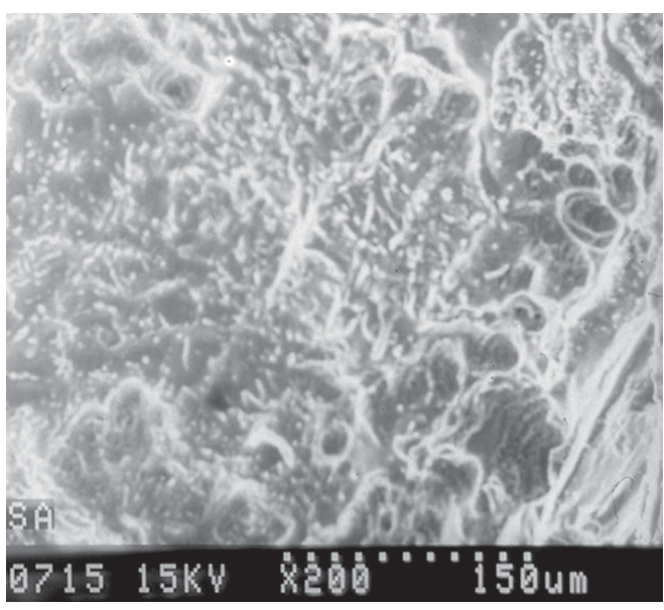

Muestra C

Figura 4. MEB de las superficies corroídas de la muestras A y C a 200X.

Fuente: (El autor)

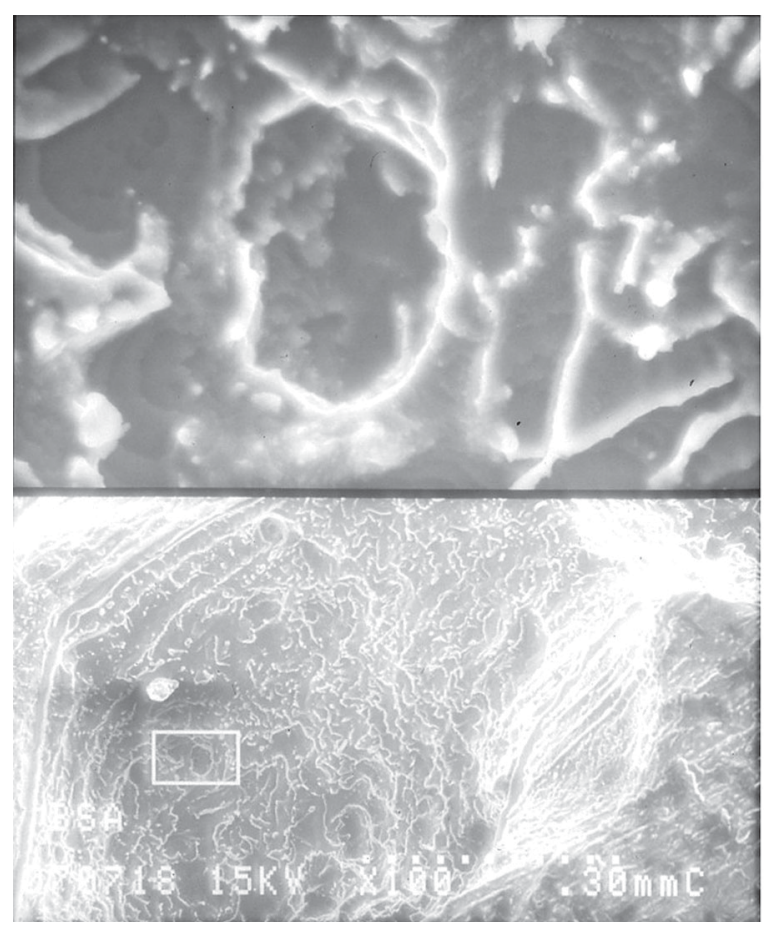

Figura 5. MEB con detalle a 500X de superficie corroída en muestra B. Fuente: (El autor) 


\section{DISCUSIÓN DE RESULTADOS}

En virtud de la importancia operativa de los ánodos galvánicos, es necesario evaluar su calidad para verificar que cumplan con las características necesarias para su aceptación. Para el desarrollo de este estudio se eligieron únicamente aleaciones de magnesio, ya que por su potencial de oxidación, son las más adecuadas para utilizarlas en sistemas de protección catódica en medios de altas resistividades, de acuerdo con lo recomendado por (Ávila \& Genescà, 1989). Esto hace que estas aleaciones figuren como las que más se vayan a utilizar en las aplicaciones que se han identificado en nuestro país.

$\mathrm{Al}$ revisar las composiciones de las aleaciones que se presentan en el Cuadro 1 , se establece que las concentraciones de los aleantes coinciden con los valores nominales solicitados; es claro que en las muestras de $\mathrm{Mg}$ de alto potencial, se encuentra menor cantidad de elementos tales como Al y Zn, y aumenta el contenido de Mn. Sin embargo, los contenidos de $\mathrm{Mg}$ aparecen en menores cantidades que las establecidas por la norma ASTM B-843, esta situación hace que sea muy importante verificar su desempeño electroquímicamente.
Para el procedimiento de medición del comportamiento electroquímico se usó una modificación al establecido en la norma ASTM G-97, debido a que las condiciones que allí se especifican requieren gran cantidad de material y extensos periodos de ensayo, resultando en una limitación, tanto por los altos costos, como por los cortos periodos que se tiene en un proceso de adquisición. Las variaciones consistieron en reducir la cantidad y dimensiones (aunque no la geometría) de las muestras anódicas y aumentar la densidad de corriente inyectada durante las pruebas galvanostáticas; las pruebas se realizaron por triplicado para tratar de reducir errores sistemáticos.

Las probetas se maquinaron a partir de una sección extraída de los bloques anódicos. La magnitud de la corriente impuesta al sistema, generó una densidad de corriente de alrededor de $1 \mathrm{~mA} / \mathrm{cm}^{2}$, la cual resulta dos órdenes de magnitud más alta que la propuesta en ASTM G-97. Esta intensidad se estableció, con base en los postulados de (Genescà, 1993), quien apunta que la densidad de corriente utilizada, corresponde a los ámbitos de corrientes máximas comúnmente utilizadas en sistemas de corriente impresa, sin que sufra un deterioro prematuro del material anódico y de forma que se obtengan desgastes significativos

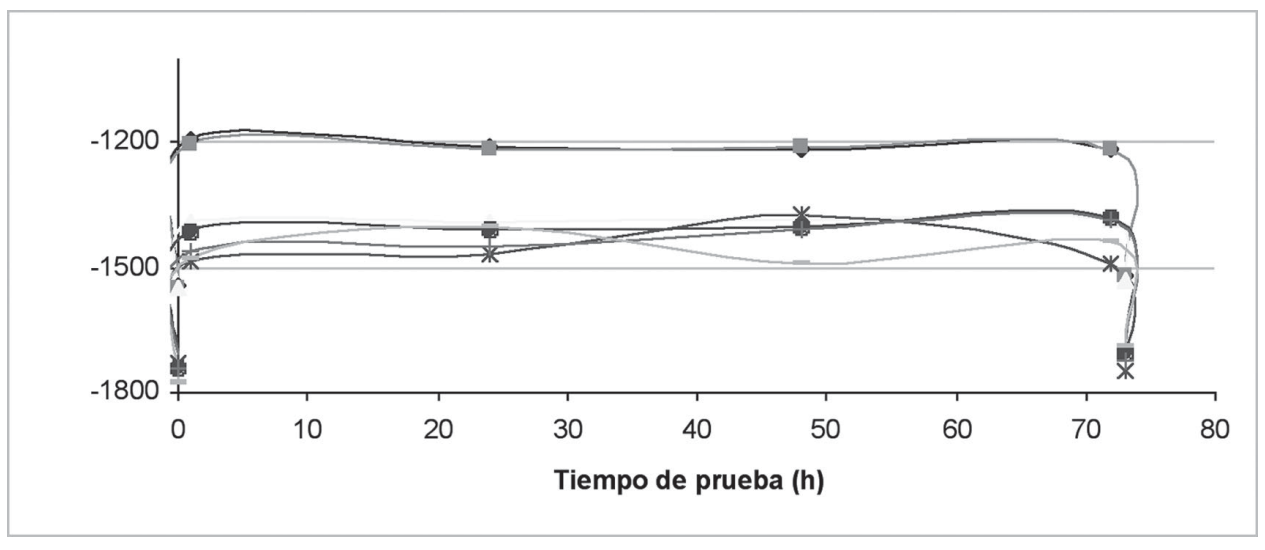

Figura 6. Potenciales de las probetas durante ensayos galvanostáticos.

Fuente: (El autor) 
del material anódico, en tiempos menores a los que se requerirían si sólo se tuviera la corriente auto generada por el sistema galvánico.

En la Figura 6 se presenta el comportamiento de los potenciales de las muestras anódicas. El primer punto de cada curva, corresponde al potencial de circuito abierto para cada una de las probetas antes de iniciar las pruebas electroquímicas, mientras que el último punto registra el potencial, pero después de concluido el periodo de inyección de corriente.

Es claro que en todos los casos los potenciales de las probetas regresaron a su potencial natural de corrosión después de sufrir la polarización anódica que aceleró su desgaste. Estos rondaron los $-1500 \mathrm{mV}$ para las probetas construidas con la muestra $\mathrm{A}$, mientras que para las de las muestras B y C, estuvieron alrededor de los $-1700 \mathrm{mV}$. Estas magnitudes coinciden con las indicadas en la literatura (ASM, 1985) para aleaciones de Mg tipo AZ63, como es el caso de la muestra A, y valores más activos para las de alto potencial, como sucede con las B y C.

Los puntos intermedios en cada una de las curvas, corresponden a los potenciales de las probetas registrados mientras se realizaba la polarización galvanostática. Estos aumentaron respecto a los de circuito abierto como era de esperarse, ya que se estaba realizando una polarización anódica sobre ellos. Por otra parte, al considerar su variación, puede observarse que estos se mantuvieron relativamente constantes en el tiempo. Este comportamiento no refleja ninguna tendencia a la pasivación, situación que podría estar indicada por un aumento significativo en su potencial.

El drenaje de corriente por unidad de masa (material disuelto) debió haber sido similar entre las probetas de una misma muestra; sin embargo, se encontraron diferencias que pueden explicarse por el hecho de que en toda aleación, hay imperfecciones o solicitaciones mecánicas en la red cristalina; lo cual hacen que sea más eficiente el drenaje de corriente en unos puntos que en otros. En el Cuadro 2, se presentan las corrientes promedio drenadas por las diferentes aleaciones estudiadas y su equivalente en términos de eficiencia. La aleación B fue la que presentó la menor eficiencia electroquímica; sin embargo, todas tienen valores muy parecidos, que coinciden con los valores típicos para ánodos comerciales de Mg citados por (Bianchetti, 2001) y (Ávila \& Genescà, 1989). La inspección microscópica de las muestras ayuda a explicar algunos de los resultados obtenidos.

En la Figura 2 se presentan las metalografías de las especies, luego de ser atacadas con solución de Nital. En la metalografía de la muestra A, la cual contiene mayor porcentaje de aleantes, se observa una cantidad considerable de manchas grises. Estas estructuras son atribuibles a una segregación de la aleación, originadas por un precipitado intergranular $\mathrm{Mg}_{17} \mathrm{Al}_{12}$, que aparece durante la fusión eutéctica al sufrir un tratamiento térmico, con el fin de reducir las microporosidades, según indican (Genescà \& Juárez, 2000); de esta forma se favorecería la disolución del material ante un proceso de corrosión.

En las fotografías restantes no se observa este fenómeno, dado que por la baja cantidad de aleantes, es difícil la formación de precipitados, teniéndose granos de disolución sólida de $\mathrm{Mg}$ prácticamente no visibles. Las probetas habían sido observadas previamente, sin ataque, donde se comprobó la presencia de incrustaciones no metálicas en todas las muestras. En todos los casos, las microestructuras coinciden con las que deberían tenerse de acuerdo con los análisis químicos obtenidos.

Luego de finalizados los ensayos electroquímicos, se inspeccionó el estado de las probetas. A simple vista, todas las muestras presentaron un depósito de productos de corrosión de contextura granulada, ligeros y no adherentes, que al limpiarlos dejan una superficie altamente rugosa, uniformemente distribuida. Al observar los productos en MEB, se aprecian gran cantidad de grumos, inclusive con fisuras y grietas, según se presenta en la Figura 3, lo que les confiere un carácter de porosos. 
Este tipo de comportamiento, muy similar en todas las muestras, es precisamente señalado por (Ávila \& Genescà, 1989), como una característica deseable para los materiales anódicos, cuyos productos ofrecen menor resistencia al intercambio iónico entre el electrolito y la superficie, dando como resultando que se logre una transferencia uniforme de carga y aumentando el rendimiento de la reacción, contrariamente a lo que sucedería cuando se generan películas adherentes.

Bajo estos productos, las superficies presentan ataque generalizado como se muestra en la Figura 4; en ninguno de los casos se encontraron indicios de corrosión por picadura o localizada; ni zonas con películas protectoras o pasivantes. En la Figura 5 se presenta una ampliación a 500X de un segmento del área atacada, donde se observa cómo la corrosión avanza formando cavernas e irregularidades sobre la superficie. Esta morfología de ataque favorece el potencial activo de las muestras, concordando con los valores de potencial obtenidos durante los ensayos electroquímicos.

\section{CONCLUSIONES}

Las aleaciones evaluadas, cumplen con los requerimientos para ser utilizados como ánodos galvánicos y obtener buenos rendimientos.

El desempeño electroquímico de los materiales ensayados corresponde con el esperado. La aleación de la muestra A fue la que mostró mayor eficiencia electroquímica.

Los registros de potencial de circuito cerrado de las aleaciones estudiadas se mantienen prácticamente constantes en el tiempo, lo que garantiza que la fuerza electromotriz para el desarrollo de las reacciones sea uniforme.

La medición del potencial de circuito abierto representa una prueba rápida y económica que ayuda a clasificar la capacidad anódica de un material; sin embargo, con este ensayo no se podría asegurar nada acerca de su desempeño electroquímico.
La fiabilidad de los resultados de un ensayo electroquímico puede aumentar si estos se llevan a cabo bajo condiciones más cercanas a las de servicio, tales como composición del electrolito, temperatura, agitación y geometría del ánodo, entre otras.

La morfología de los productos de corrosión indican que los ánodos no sufrirán una pasivación prematura, por cuanto se observa la formación de productos de corrosión de forma generalizada, porosos y no adherentes.

La estructura metalográfica de las aleaciones analizadas corresponden con las reportadas en la literatura para este tipo de materiales. Las microestructuras revelan que todas las muestras fueron sometidas a un tratamiento térmico, que poseen imperfecciones e incrustaciones en su red cristalina; sin embargo, no se observaron microagrietamientos que pudieran contribuir a una reducción adicional en su rendimiento.

Un análisis integral para evaluación de ánodos requiere contar con equipo adecuado, material suficiente y periodos de experimentación relativamente extensos. En la medida de lo posible, se debe realizar un muestreo fundamentado estadísticamente.

\section{RECOMENDACIONES}

Con el fin de establecer un mecanismo para el control de calidad de aleaciones de magnesio, se sugiere evaluar el material que se desea utilizar, considerando los aspectos que se detallan en el formulario que se presenta en el Anexo A.

Se recomienda establecer procedimientos similares para evaluación del desempeño de otras aleaciones tales como $\mathrm{Al}$ y $\mathrm{Zn}$.

\section{AGRADECIMIENTO}

Se agradece al Consejo Nacional para Investigaciones Científicas y Tecnológicas (CONICIT) por su apoyo en el desarrollo de este trabajo. 


\section{REFERENCIAS BIBLIOGRÁFICAS}

ASM (American Society for Metals). (1985). Metals Handbook: metallography and microestructures. Vol. 9. Ohio: ASM Handbook Committee. 425-434.

ASTM (American Society Testing Materials). (2002). Annual Book of Standards. Vol 03.02. B-843 Standard specification for magnesium alloy anodesfor cathodic protection. Philadelphia, EEUU: ASTM.

ASTM (American Society Testing Materials). (2002). Annual Book of Standards. Vol 03.02. G-97 Standard test method for laboratory evaluation of magnesium sacrificial anode test specimens for undergroundapplications. Philadelphia, EEUU: ASTM.

Ávila, J. \& Genescà, J. (1989). Más allá del herrumbre II. México D.F.: Fondo de Cultura Económica.

Bianchetti, R. (2001). Peabody's control of pipeline corrosion. ( $2^{\mathrm{a}}$ ed.). Houston, EEUU: NACE Press.

Genescà, J. (1993, junio). Evaluación electroquímica de ánodos galvánicos. En: Primer Seminario Internacional IMICORR'93. Veracruz, México.

Genescà, J. \& Juárez, J. (2000). Development and testing of galvanic anodes for cathodic protection. Contributions to Science, 1(3), 331-343.

Hernández, M., Genescà, J. \& Uruchurtu, J. (2002). Electrochemical characterization of an Al-Zn-In galvanic anode (Paper
02022). En: Seminario Corrosion 2002. Houston, EEUU.

González, J. (1989). Control de la corrosión: estudio y medida por técnicas electroquímicas. Madrid, España: Consejo Superior de Investigaciones Científicas. CENIM.

Miranda, J. \& Marrero, R. (1997). Campos de aplicación de la protección catódica. Madrid, España: CUJAE-WWI Procat.

NACE (National Association of Corrosion Engineers). (2003) NACE Standards. RP-0169 Control of external corrosion on underground or submerged metallic piping systems. Houston, EEUU: NACE Internacional.

Von Baeckmann, W., Schwenk, W \& Prinz, W. (1997). Handbook of cathodic protection. ( $3^{\text {a }}$ ed.). Houston, EEUU: Gulf Publishing Company.

Zavala, A., Puente, I., Rodríguez, C. \& Genescà, J. (2002). Evaluación de ánodos de aluminio mediante los procedimientos DNV RP B401 y NACE TM 190-98. Afinidad 59(497): 9-16.

\section{SOBRE EL AUTOR}

\section{Mario Alberto Vega León}

Ingeniero Químico, Master en Ingeniería Química con énfasis en procesamiento de materiales.

Centro de Investigación de Corrosión, del Instituto Costarricense de Electricidad

Teléfono: $220-5164$

Facsímil: 297-4070

Correo electrónico:mvegal@ice.go.cr 


\section{ANEXO A}

Ficha de control de aceptación de ánodos de magnesio

Identificación física del ánodo:

Fecha: 1

Presenta certificado de calidad:

Sí $\square \quad$ No $\square$

El certificado de calidad contiene la siguiente información:

\begin{tabular}{|c|c|c|}
\hline Razón social del fabricante: & & No $\square$ \\
\hline Fecha de fabricación: & Sí & No \\
\hline Especificación de la aleación: & Sí & No \\
\hline Composición química del ánodo: & Sí & No \\
\hline Peso bruto: & Sí & No \\
\hline Peso neto: & Sí & No \\
\hline Potencial de circuito abierto: & Sí & No \\
\hline Capacidad de corriente real: $(\mathrm{Ah} / \mathrm{kg})$ & Sí & No \\
\hline
\end{tabular}

Inspección física:

Peso: $\mathrm{kg}$

Dimensiones:

Presencia de grietas o fracturas en el cuerpo del ánodo

Sí $\square \quad$ No

Presencia de partículas o inclusiones en la superficie del ánodo

Sí $\square \quad$ No $\square$

El alma del ánodo se encuentra expuesta sólo para los puntos de apoyo o conexión eléctrica:

Sí $\quad$ No $\square$

Presenta indicios de productos de corrosión de color blanco

Sí $\square \quad$ No $\square$

La conexión del cable eléctrico (si lo tuviera) se observa firme $\quad$ Sí $\quad \begin{array}{lll}\text { No }\end{array}$

Análisis químico:

Reporte de análisis químico de los siguientes componentes principales:

\begin{tabular}{llllllll}
\hline & $\mathrm{Mg}$ & $\mathrm{Al}$ & $\mathrm{Zn}$ & $\mathrm{Mn}$ & $\mathrm{Cu}$ & $\mathrm{Fe}$ & $\mathrm{Ni}$ \\
\hline \% peso & & & & & & & \\
\hline
\end{tabular}

Comportamiento electroquímico:

(Se llevará a cabo de acuerdo con los lineamientos de la norma ASTM G-97, en caso de que no se disponga del material y tiempo suficiente, se evaluará empleando geometrías similares y densidades de corriente de $1 \mathrm{~mA} / \mathrm{cm}^{2}$ aprox.)

- Reporte de potenciales de circuito abierto y cerrado:

- Reporte de capacidad de corriente: $\mathrm{Ah} / \mathrm{kg}$

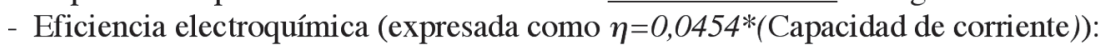

Observaciones:

Figura A.1. Formulario de inspección 
